\title{
Antibiotic Susceptibility Pattern in Clinical Isolates of Pseudomonas aeruginosa from a Tertiary Care Hospital of Tripura
}

\author{
Jayanta Debnath ${ }^{1}$, Anup Saha ${ }^{1}{ }^{*}$, Pradip Kumar Das $^{1}$, \\ Niladri Sekhar Das ${ }^{1}$ and Soma Saha ${ }^{2}$
}

${ }^{1}$ Department of Microbiology, ${ }^{2}$ Department of Medicine, Tripura Medical College \& Dr. B.R. Ambedkar Memorial Teaching Hospital, Agartala, Tripura, India

*Corresponding author

\section{Keywords \\ Pseudomonas aeruginosa, Antibiotic susceptibility, Treatment \\ Article Info \\ Accepted: \\ 04 February 2019 \\ Available Online: \\ 10 March 2019}

\section{A B S T R A C T}

Pseudomonas aeruginosa are established pathogens predominantly in various nosocomial infections which are often life threatening due to limited therapeutic options. The present study was undertaken to analyze the antibiotic susceptibility pattern in the clinical isolates of Pseudomonas aeruginosa, so as to establish the current therapeutic options available for treatment in this geographical area of North East India. The study was conducted on 150 strains of Pseudomonas aeruginosa isolated from various clinical samples. Isolation and confirmation of the organism in culture was performed using standard microbiological techniques. The antibiotic susceptibility testing was performed by Kirby Bauer Disc diffusion method. Out of all clinical samples 6.6\% were identified to be Pseudomonas aeruginosa and $69.4 \%$ were elderly male patients. The highest numbers $(62.7 \%)$ of isolates were from pulmonary samples like sputum, Endotracheal aspirate and Bronchoalveolar lavage, followed by urine $(25.3 \%) .10 .7 \%$ isolates were resistant to Imipenem, followed by Meropenem (12.7\%), Piperacillin Tazobactum (14\%) and Piperacillin (28.7\%). Higher level of resistance was observed with Nitrofurantoin(71.1\%), Gentamicin (60.7\%), Amikacin (42\%), Ciprofloxacin (46\%) and Levofloxacin (42\%). A total of 43(28.7\%) isolates were Multi-Drug resistant. In our hospital, the antibiotic of choice for treatment of infections due to Pseudomonas aeruginosa could be Piperacillin Tazobactum. Emphasis on strict adherence to hospital infection control guidelines and antibiotic policy is also recommended as most of the isolates were recovered from hospitalized patients.

\section{Introduction}

Pseudomonas aeruginosa are gram negative, aerobic, non-fermentative bacilli, widely distributed in nature and hospital environment. It is responsible for $10-20 \%$ of nosocomial infections (Carmeli et al., 1999). They are established pathogens in nosocomial
Pneumonia, Urinary tract infections, skin and soft tissue infections, burns, injuries, septicemia and infections in immunocompromised conditions.

The infections caused by Pseudomonas aeruginosa are often life threatening due to limited therapeutic options, owing to the 
constitutive low level of susceptibility to several antibiotics and multiple genetic mechanisms of resistance (Babay, 2007). Their resistance to antibiotics may be due to mutation in chromosomal genes which regulate the resistance genes and acquisition of additional genes from other organisms or environment via plasmids, transposons and bacteriophages. Increase in the prevalence of Multi-Drug Resistant (MDR) strains of Pseudomonas aeruginosa has been reported worldwide, complicating decisions on antibiotic policy and its relation to high morbidity and mortality (Babay, 2007; Ergin and Mutlu, 1999). The variations in antibiotic susceptibility exists in different geographical locations and periods, due to difference in pattern of prescribing habits, for which periodic analysis of antibiotic susceptibility pattern is essential to know the susceptible therapeutic options available for treatment. The objective of this study was to analyze the antibiotic susceptibility pattern in the clinical isolates of Pseudomonas aeruginosa, so as to establish the current therapeutic options available for treatment.

\section{Materials and Methods}

The prospective study was conducted in the Microbiology Department of Tripura Medical College \& Dr. BR Ambedkar Memorial Hospital, a tertiary care centre of the North Eastern State of Tripura. The proposal for the study was approved by the Institutional Human Ethics Committee. The study was conducted on 150 strains of Pseudomonas aeruginosa isolated from various clinical samples during a period of one year six months from July 2014 to December 2015. The particulars and clinical data of the patients were recorded simultaneously.

In the laboratory all collected samples were cultured aerobically on Blood agar and MacConkey agar media plates at $37^{\circ} \mathrm{C}$ for 24 hours. Blood specimen were cultured in Brain Heart Infusion Broth and subsequently subcultured in Blood agar and MacConkey agar plates. Suspected Non-Lactose Fermenting colonies of Pseudomonas aeruginosa were identified using colony morphology, motility testing, Grams reaction and biochemical tests indicating positive oxidase test, alkaline slant in Triple Sugar Iron agar medium, negative Indole production test, positive citrate utilization test and positive nitrate reduction test. Definitive identification of Pseudomonas aeruginosa included identifying the production of the blue green pigment pyocyanin and its ability to grow at $42^{\circ} \mathrm{C}$ (Collee et al., 2006). Antibiotic susceptibility testing was performed against AntiPseudomonal antibiotics by modified Kirby Bauer Disc diffusion method conforming to the CLSI guidelines. (CLSI, 2014) AntiPseudomonal antibiotics used for susceptibility testing were from the classes of Ureidopenicillins, Cephalosporins, Carbapenems, Aminoglycosides and Fluoroquinolones. For Quality control Pseudomonas aeruginosa ATCC 27853 strain was used. The Multi-Drug Resistant (MDR) strains of Pseudomonas aeruginosa were identified by the criteria that those which are resistant to three or more classes of AntiPseudomonal antibiotics (Magiorakos, 2011).

\section{Results and Discussion}

The data collected for a period of 18 months reveals that, out of 7368 samples, culture was positive in 2274 cases and 150(6.6\%) isolates were identified to be Pseudomonas aeruginosa. The majority of the patients were males $(56 \%)$ and of elderly age group of more than 60 years $(48 \%)$ as shown in the Table 1 (Fig. 1).

Varied spectrum of the lower respiratory tract infections with Chronic obstructive pulmonary disease were the most common clinical cases 
in which the organisms were isolated. The isolates of Pseudomonas aeruginosa were most commonly identified from lower respiratory tract secretions $94(62.7 \%)$ in specimen like sputum, Broncho-alveolar lavage and Endotracheal aspirates, followed by urine $38(25.3 \%)$ as mentioned in the Table 2 (Fig. 2).

Among the Beta-Lactams tested, the most effective agent was Imipenem 134(89.3\%) followed by Meropenem 131(87.3\%), Piperacillin 107(71.3\%), Cefepime 98(65.3\%), Ceftazidime 96(64\%). The susceptibility results of combination of Beta-Lactams and Beta-Lactamase inhibitors tested were Piperacillin-tazobactum 129(86\%) and Cefoperazone-sulbactum 103(68.7\%). Among the Aminoglycosides, Netilmicin showed considerable sensitivity of 93(62\%), followed by Amikacin 87(58\%). Only $81(54 \%)$ isolates were sensitive to Ciprofloxacin and $87(58 \%)$ to Levofloxacin. Nitrofurantoin was additionally tested against the 38 urine isolates of Pseudomonas aeruginosa, in which only $11(28.9 \%)$ of the isolates were susceptible. The observation is depicted in Table 3 (Fig. $3)$. A total of $43(28.7 \%)$ isolates were MultiDrug resistant, i.e. resistant to three or more antibiotic classes.

In the present study, Pseudomonas aeruginosa were isolated in $6.6 \%$ of the culture positive cases, which can be compared with reports from Odisha and Andhra Pradesh stating $8.43 \%$ and $9.3 \%$ prevalence rate respectively (Pathi et al., 2013; Srinivas et al., 2012). The prevalence rate may vary depending upon type of clinical specimen, status of healthcare centre, demographic profiles and geographical location (Dash et al., 2014). A prevalence as low as $2.1 \%$ has been reported from Nigeria, whereas a high prevalence rate of $32.1 \%$ was reported from Gujarat (Okon et al., 2010; Rajat et al., 2012). In our observation, 56\% of the patients were males and $48 \%$ of the patients belonged to age more than 60 years. Out of the total number of elderly patients, 69.4\% were elderly male patients (Fig. 1). Other studies also reported similar observation, ranging from $62.5 \%$ to $71 \%$ of elderly male patients being infected with Pseudomonas aeruginosa (Javiya et al., 2008; Mayank et al., 2009)

In the present study, maximum number $(62.7 \%)$ of isolates were from pulmonary samples like sputum, Endotracheal aspirate and Bronchoalveolar lavage, followed by urine $(25.3 \%)$ and pus (7.3\%) (Fig. 2). This observation is different from most of the reported studies, in which isolation of Pseudomonas aeruginosa from pus and urine samples predominates over sputum (Dash et al., 2014; Okon et al., 2010; Mohanasoundaram, 2011) A study from Uttar Pradesh reported that highest number of isolates $(53.89 \%)$ of Pseudomonas aeruginosa were from pulmonary samples. (Prakash et al., 2014) A study from Kathmandu reported that maximum number of isolates of Pseudomonas aeruginosa were from urine and sputum (36.3\% each). (Shrestha et al., 2016) The high rate of isolation of Pseudomonas aeruginosa from pulmonary samples during our study period may be due to a possible outbreak of infection in General Medicine ward and Intensive Care Unit, as most of the admitted patients were previously diagnosed as Chronic Obstructive Pulmonary Disease.

On analysis of Antibiotic susceptibility pattern, we observed that $10.7 \%$ isolates were resistant to Imipenem, followed by Meropenem (12.7\%), Piperacillin Tazobactum (14\%) and Piperacillin (28.7\%). Higher level of resistance was observed with Gentamicin (60.7\%), Amikacin (42\%), Ciprofloxacin (46\%), Levofloxacin (42\%) and Nitrofurantoin in urine isolates $(71.1 \%)$. The isolates did not reveal an acceptable level of sensitivity for therapeutic use to other tested 
antibiotics like Cefepime, Ceftazidime, Netilmicin and Cefoperazone sulbactum (Fig. $3)$. In our centre, keeping the Carbapenems as "reserve drugs", the Ureidopenicillins preferably in combination with a BetaLactamase inhibitor like Piperacillin Tazobactum may be considered as drug of choice for treatment of patients suffering from infections due to Pseudomonas aeruginosa. Concurrent administration of a BetaLactamase inhibitor markedly expands the spectrum of activity of acid resistant Penicillins like Piperacillin and Ticarcillin. The dose and incidence of toxicity also gets subsequently reduced with Ureidopenicillins.

Similar observation was reported in a recent study from Odisha, stating Imipenem, Meropenem and Piperacillin Tazobactum to be the most effective drugs against infections due to Pseudomonas aeruginosa, exhibiting resistance rates as low as $6.4 \%, 8 \%$ and $11.3 \%$ respectively. However, in their observation the isolates were less resistant to Aminoglycosides and Fluoroquinolones, unlike our report (Dash et al., 2014). This might be due to widespread administration of Aminoglycosides and Fluoroquinolones in hospital and community for treatment of infections and surgical prophylaxis in medical practice in this region.

In the present study, $28.7 \%$ isolates of Pseudomonas aeruginosa were Multi-Drug Resistant, which implies resistance to three or more antibiotic classes. Observations reported from Uttar Pradesh and Pakistan are quite similar as ours, stating $31.7 \%$ and $22.7 \%$ rate of isolation of Multi-Drug Resistant Pseudomonas aeruginosa respectively (Prakash et al., 2014; Gill et al., 2011). MultiDrug Resistant Pseudomonas aeruginosa elaborates inactivating enzymes which can be chromosomally encoded or plasmid mediated, that make Beta-Lactams and Carbapenems ineffective, such as Extended Spectrum Beta Lactamases and Metallo Beta Lactamases (Vahdani et al., 2012).

Table.1 Proportions of Age-Group of patients in relation to sex and isolates of Pseudomonas aeruginosa

\begin{tabular}{|c|c|c|c|}
\hline $\begin{array}{l}\text { Age - Group of } \\
\text { patients }\end{array}$ & $\begin{array}{c}\text { Number of Isolates } \\
\mathbf{N}(\%)\end{array}$ & $\begin{array}{l}\text { Males } \\
\mathbf{N}(\%)\end{array}$ & $\begin{array}{c}\text { Females } \\
\mathbf{N}(\%)\end{array}$ \\
\hline 0 - 15 years & $05(3.3 \%)$ & $02(40 \%)$ & $03(60 \%)$ \\
\hline $16-30$ years & $11(7.3 \%)$ & $04(36.4 \%)$ & $07(63.6 \%)$ \\
\hline 31 - 45 years & $27(18 \%)$ & $10(37 \%)$ & $17(63 \%)$ \\
\hline $46-60$ years & $35(23.3 \%)$ & $18(51.4 \%)$ & $17(48.6 \%)$ \\
\hline$>60$ years & $72(48 \%)$ & $50(69.4 \%)$ & $22(30.6 \%)$ \\
\hline TOTAL & 150 & $84(56 \%)$ & $66(44 \%)$ \\
\hline
\end{tabular}

Table.2 Proportion of Isolates from various clinical specimen

\begin{tabular}{|l|c|c|}
\hline Specimen & $\begin{array}{c}\text { Isolates } \\
\mathbf{N = 1 5 0}\end{array}$ & $\begin{array}{c}\text { Proportion } \\
(\mathbf{\%})\end{array}$ \\
\hline Pulmonary samples & 94 & $62.7 \%$ \\
\hline Urine & 38 & $25.3 \%$ \\
\hline Pus & 11 & $7.3 \%$ \\
\hline Blood & 07 & $4.6 \%$ \\
\hline
\end{tabular}


Table.3 In vitro susceptibility pattern of Pseudomonas aeruginosa isolates

\begin{tabular}{|l|c|c|}
\hline $\begin{array}{l}\text { Antibiotic (Disk concentration in } \\
\boldsymbol{\mu g})\end{array}$ & $\begin{array}{l}\text { Proportion of susceptible } \\
\text { isolates }[\mathbf{N}(\mathbf{\%})]\end{array}$ & $\begin{array}{l}\text { Proportion of resistant } \\
\text { isolates [N(\%)] }\end{array}$ \\
\hline Imipenem (10) & $134(89.3 \%)$ & $16(10.7 \%)$ \\
\hline Meropenem (10) & $131(87.3 \%)$ & $19(12.7 \%)$ \\
\hline Piperacillin-tazobactum (100/10) & $129(86 \%)$ & $21(14 \%)$ \\
\hline Piperacillin (100) & $107(71.3 \%)$ & $43(28.7 \%)$ \\
\hline Cefoperazone-sulbactum (75/30) & $103(68.7 \%)$ & $47(31.3 \%)$ \\
\hline Cefepime (30) & $98(65.3 \%)$ & $52(34.7 \%)$ \\
\hline Ceftazidime (30) & $96(64 \%)$ & $54(36 \%)$ \\
\hline Amikacin (30) & $87(58 \%)$ & $63(42 \%)$ \\
\hline Gentamicin (10) & $59(39.3 \%)$ & $91(60.7 \%)$ \\
\hline Netilmicin (30) & $93(62 \%)$ & $57(38 \%)$ \\
\hline Levofloxacin (5) & $87(58 \%)$ & $63(42 \%)$ \\
\hline Ciprofloxacin (5) & $81(54 \%)$ & $69(46 \%)$ \\
\hline $\begin{array}{l}\text { Nitrofurantoin (300) } \\
\text { (For Urine isolates (n=38)) }\end{array}$ & $11(28.9 \%)$ & $27(71.1 \%)$ \\
\hline
\end{tabular}

Fig.1 Proportion of age group of patients in relation to sex and isolates of Pseudomonas aeruginosa

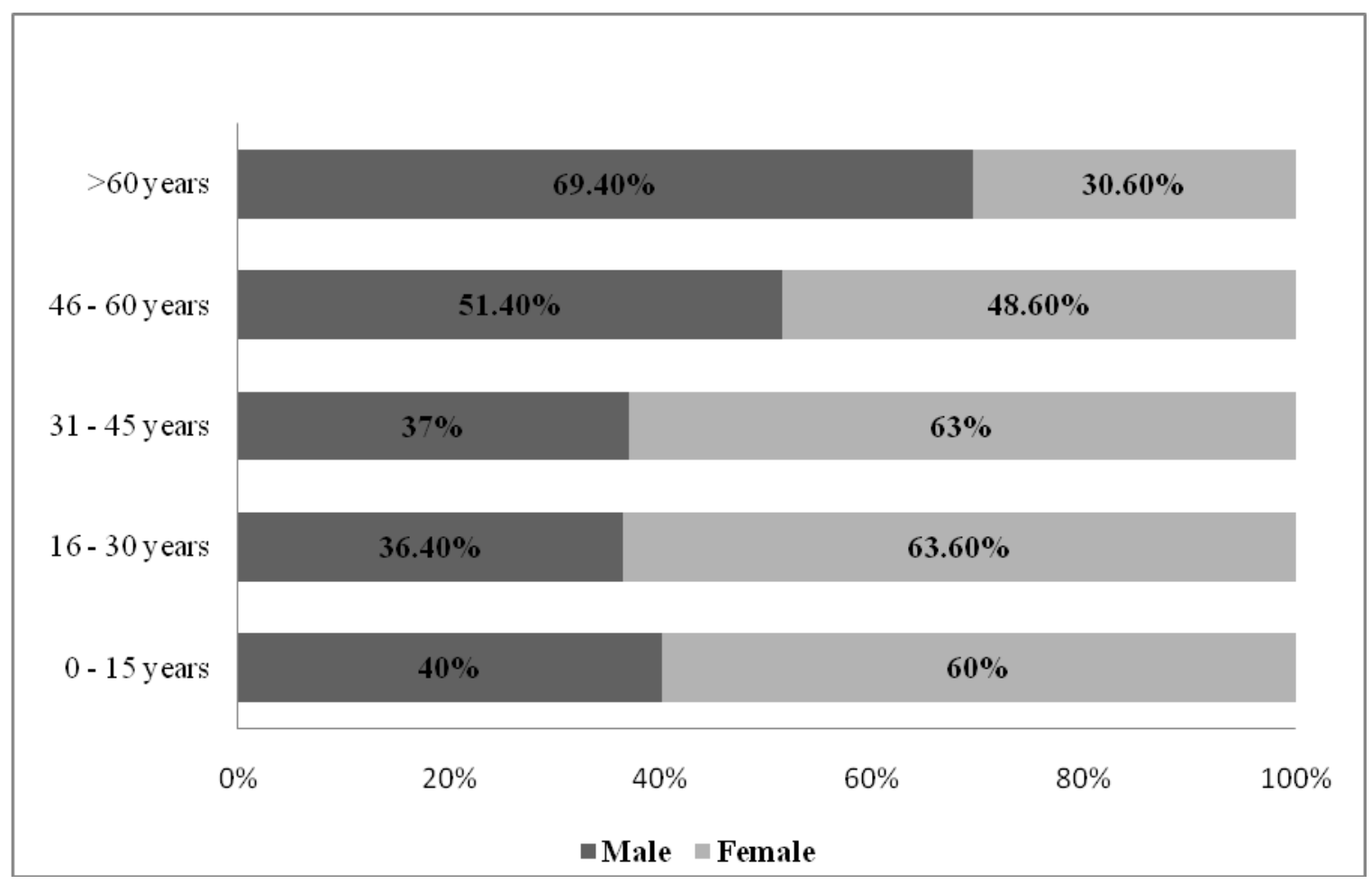


Fig.2 Proportion of isolates from various clinical samples

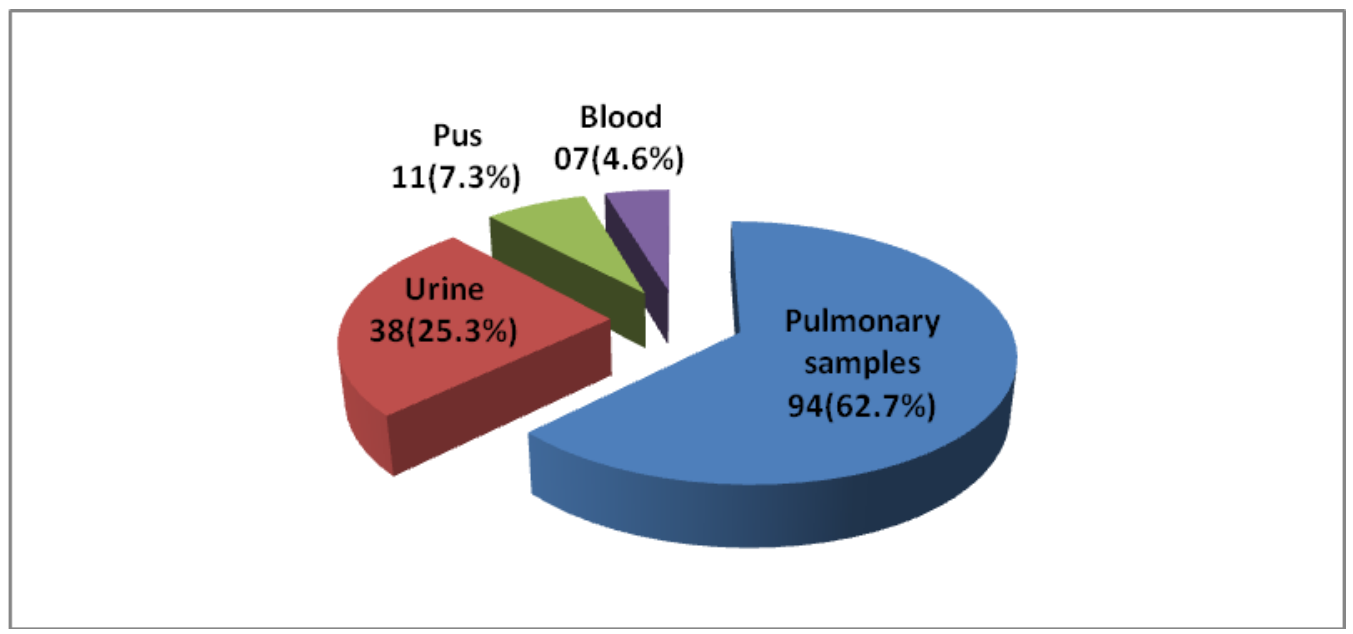

Fig.3 In vitro antibiotic susceptibility pattern of the isolated Pseudomonas aeruginosa

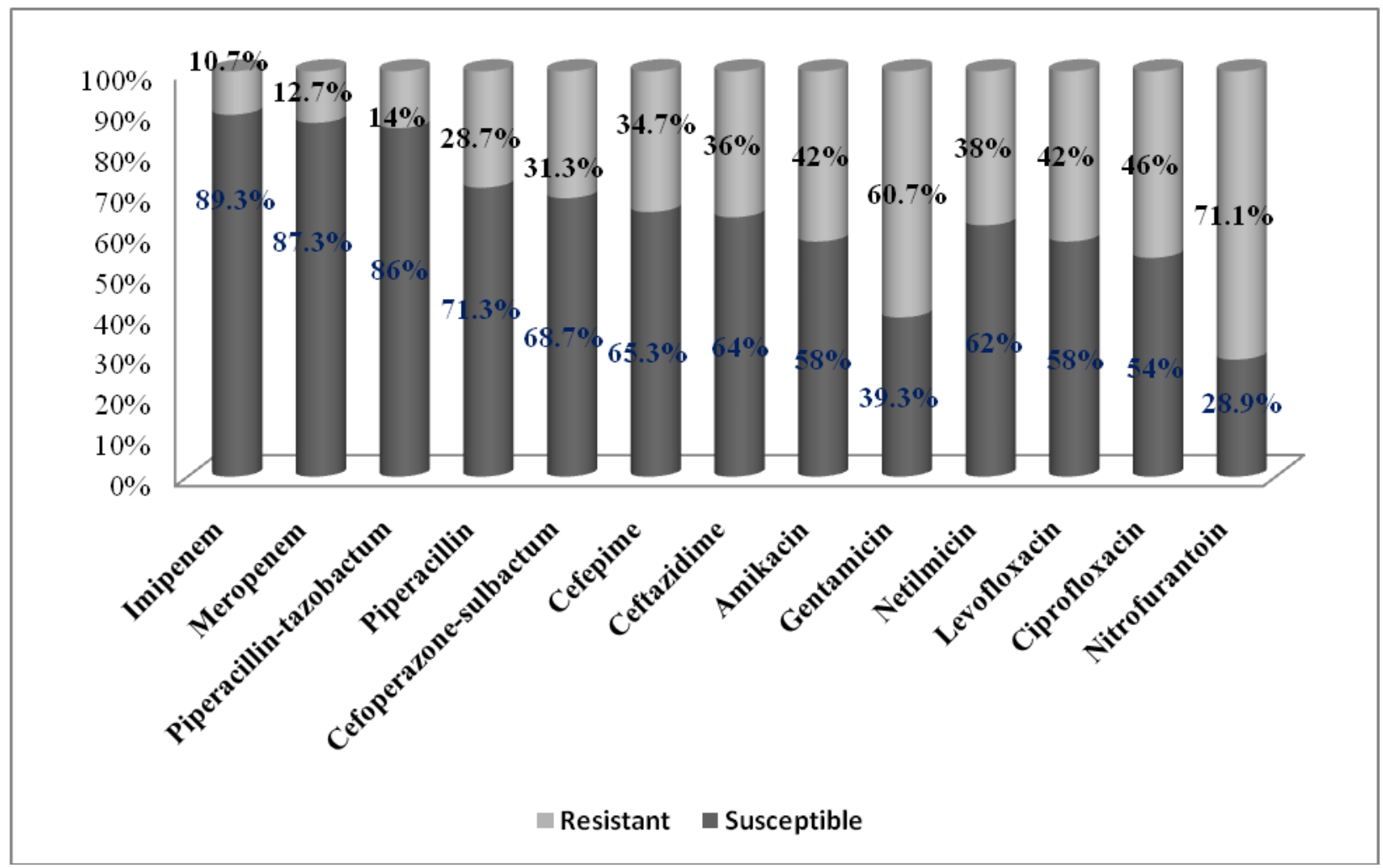

The increasing trend of drug resistance exhibited by Pseudomonas aeruginosa is an established fact, which can be due to irrational use of broad spectrum antibiotics and its unique feature to acquire resistance due to low permeability of the cell wall, production of inducible Cephalosporinases, an active efflux with a poor affinity to target sites. (Al-Tawfiq, 2007).

In conclusion, in our healthcare setting, Piperacillin Tazobactum can be considered as 
the appropriate antibiotic for treating infections due to Pseudomonas aeruginosa. As, majority of the isolates were recovered from hospitalized patients, we need to emphasize upon strict adherence to hospital infection control guidelines and antibiotic policy. Periodic antimicrobial surveillance is also recommended to keep a track on the development and spread of Multi-Drug Resistant Pseudomonas aeruginosa.

\section{Acknowledgement}

We would like to thank all technical staff and especially Mr. Sumit Debnath, Laboratory Technician, for extending all necessary support in the Department of Microbiology.

\section{References}

Al-Tawfiq JA., 2007. Occurrence and antimicrobial resistance pattern of inpatient and outpatient isolates of Pseudomonas aeruginosa in a Saudi Arabian hospital: 1998-2003. Int J Inf Dis.; 11: 109-114.

Babay HA., 2007. Antimicrobial resistance among clinical isolates of Pseudomonas aeruginosa from patients in a teaching hospital, Riyadh, Saudi Arabia, 20012005. Jpn J Infect Dis.; 60: 123-125.

Carmeli YN, Troillet G, Eliopoulos GM, Samore MH., 1999. Emergence of antibiotic resistant Pseudomonas aeruginosa: Comparison of risks associated with different antipseudomonal agents. Antimicrob Agents Chemother.; 3: 1379-1382.

CLSI, 2014. Performance standards for antimicrobial susceptibility testing: Twenty fourth informational supplement. CLSI document M100 S24. Clinical and Laboratory Standards Institute, Wayne; PA: USA.

Collee JG, Miles RS, Watt B., 2006. Tests for identification of Bacteria. In: Collee JG,
Fraser AG, Marmion BP, Simmons A, editors. Mackie and McCartney Practical Medical Microbiology. $14^{\text {th }}$ ed. Singapore: Churchill Livingstone; $p$. 136-149.

Dash M, Padhi S, Narasimham MV, Pattnaik S., 2014. Antimicrobial resistance pattern of Pseudomonas aeruginosa isolated from various clinical samples in a tertiary care hospital, South Odisha, India. Saudi J Health Sci.; 3: 15-19.

Ergin C, Mutlu G., 1999. Clinical distribution and antibiotic resistance of Pseudomonas species. East J Med.; 4: 65-69.

Gill MM, Usman J, Kaleem F, Hassan A, Khalid A, Anjum R, Fahim Q., 2011. Frequency and antibiogram of multidrug resistant Pseudomonas aeruginosa. J Coll Physicians Surg Pak.; 21: 531534.

Javiya VA, Ghatak SB, Patel KR, Patel JA., 2008. Antibiotic susceptibility patterns of Pseudomonas aeruginosa at a tertiary care hospital in Gujarat, India. Indian $J$ Pharmacol.; 40: 230-234.

Magiorakos AP., 2011. Multidrug Resistant(MDR), Extensively Drug Resistant (XDR) and Pandrug Resistant (PDR) bacteria in healthcare settings. Expert proposal for a standardized international terminology. Available online at www.escmid.org.

Mayank D, Anshuman M, Singh RK, Afzal A, Baronia AK, Prasad KN., 2009. Nosocomial cross-transmission of Pseudomonas aeruginosa between patients in a tertiary intensive care unit. Indian J Pathol Microbiol.; 52: 509513.

Mohanasoundaram KM., 2011. The antimicrobial resistance pattern in the clinical isolates of Pseudomonas aeruginosa in a tertiary care hospital; 2008-2010 (A 3 yr study). J Clin Diagn Res.; 5: 491-494. 
Okon KO, Agukwe PC, Oladosu W, Balogun ST, Uba A. 2010. Antibiotic resistance pattern of Pseudomonas aeruginosa isolated from clinical specimens in a tertiary care hospital in Northeastren Nigeria. Internet $J$ Microbiol.; 8: 1-6.

Pathi B, Mishra SN, Panigrahi K, Poddar N, Lenka PR, Mallick B, Pattanik D, Jena J., 2013. Prevalence and antibiogram pattern of Pseudomonas aeruginosa in a tertiary care hospital from Odisha, India. Transw Med J.; 1: 77-80.

Prakash V, Mishra PP, Premi HK, Walia A, Dhawan S, Kumar A.,2014. Increasing incidence of multidrug resistant Pseudomonas aeruginosa in patients of a tertiary care hospital. Int $J$ Res Med Sci.; 2: 1302-1306.

Rajat RM, Ninama GL, Mistry K, Parmar R, Patel K, Vegad MM.,2012. Antibiotic resistance pattern in Pseudomonas aeruginosa species isolated at a tertiary care Hospital, Ahmedabad. Natl J Med Res.; 2: 156-159.

Shrestha S, Amatya R, Adhikari RP., 2016. Prevalence and antibiogram of Pseudomonas aeruginosa isolated from clinical specimens in a Teaching Hospital, Kathmandu. Int J Infect Dis.; 45: 115-116.

Srinivas B, Devi DL, Rao BN., 2012. A Prospective study of Pseudomonas aeruginosa and its Antibiogram in a Teaching Hospital of Rural setup. $J$ Pharm Biomed Sci.; 22: 1-4.

Vahdani M, Azimi L, Asghari B, Bazmi F, Ra stegar LA. 2012. Phenotypic screening of extended-spectrum $\beta$-lactamase and metallo-ß-lactamase in multidrugresistant Pseudomonas aeruginosa from infected burns. Ann Burns Fire Disasters.; 25: 78-81.

\section{How to cite this article:}

Jayanta Debnath, Anup Saha, Pradip Kumar Das, Niladri Sekhar Das and Soma Saha. 2019. Antibiotic Susceptibility Pattern in Clinical Isolates of Pseudomonas aeruginosa from a Tertiary Care Hospital of Tripura. Int.J.Curr.Microbiol.App.Sci. 8(03): 291-298. doi: https://doi.org/10.20546/ijcmas.2019.803.036 